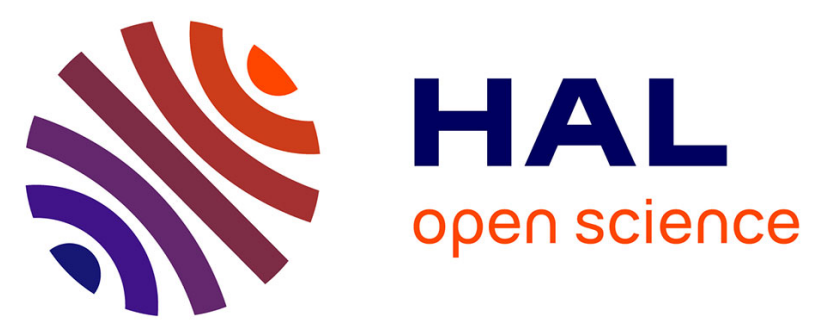

\title{
Le parvis et la porte du IVe pylône: considérations sur une chapelle et des obélisques
}

Luc Gabolde

\section{To cite this version:}

Luc Gabolde. Le parvis et la porte du IVe pylône: considérations sur une chapelle et des obélisques. Christiane Zivie-Coche et Ivan Guermeur. Parcourir l'éternité, Hommages à Jean Yoyotte, 156, Brepols Publishers, pp.459-481, 2012, Bibliothèque de l'École des Hautes Études, Section des Sciences religieuses, 978-2-503-54756-5. 10.1484/M.BEHE-EB.4.00435 . halshs-00751815v3

\section{HAL Id: halshs-00751815 \\ https://shs.hal.science/halshs-00751815v3}

Submitted on 2 Nov 2018

HAL is a multi-disciplinary open access archive for the deposit and dissemination of scientific research documents, whether they are published or not. The documents may come from teaching and research institutions in France or abroad, or from public or private research centers.
L'archive ouverte pluridisciplinaire HAL, est destinée au dépôt et à la diffusion de documents scientifiques de niveau recherche, publiés ou non, émanant des établissements d'enseignement et de recherche français ou étrangers, des laboratoires publics ou privés. 
This the author's personnal manuscript as Brepol's editions have forbiden the online publication of their own version published in Chr. Zivie-Coche, I. Guermeur (éds), Parcourir l'éternité, Hommages Jean Yoyotte, Bibliothèque de l'École des Hautes Études, Section des Sciences religieuses, Turnhout, 2012. It is more a plus than a minus as the editor's version had badly distorded the fig. $12 \& 13$ p. 474 , betraying so the author's argumentation. The editor's corrupted version is still accessible through the editor's site, though the author has expressed his disagreement, advocating his moral right (Article 1, § 2 of the 30th june 1994's law of the Belgian legislation dealing with the author's rights).

\title{
LE PARVIS ET LA PORTE DU IV ${ }^{\mathrm{e}}$ PYLONE :
} CONSIDERATIONS SUR UNE CHAPELLE ET DES OBELISQUES

\author{
LUC GABOLDE *
}

Douter de tout ou tout croire sont deux solutions également commodes qui nous dispensent de réfléchir

Henri POINCARE

Dans un article paru en 1953, Jean Yoyotte avait brillamment décortiqué les phases d'évolution du parvis du $\mathrm{IV}^{\mathrm{e}}$ pylône et plus particulièrement les aménagements qui avaient concerné sa porte monumentale. C'est un devoir autant qu'un plaisir que de prolonger les analyses de ce grand savant en revenant sur les étapes qui concernèrent l'évolution de ce secteur et les différentes interprétations qui en ont été depuis proposées.

\section{Le parvis du IV pylône, état de la question}

1.1. Convoquant des représentations de tombes jusque-là mal interprétées et des blocs de la cour à péristyle de Thoutmosis IV exhumés des fondations du IIIe pylône, J. Yoyotte montrait donc que l'avant-porte du IVe pylône, pourvue d'un dais à deux colonnes en bois dorées, avait été dressée par Thoutmosis IV avant d'être restaurée par Chabaka ${ }^{1}$, livrant ainsi un beau prolongement à l'étude des inscriptions éthiopiennes qu'avait menée peu de temps auparavant J. Leclant ${ }^{2}$. Leurs conclusions étaient entérinées par P. Barguet ${ }^{3}$. Les fouilles de J. Lauffray et J. Vérité, reprenant des sondages d'H. Chevrier permirent d'en savoir plus sur le passage du III ${ }^{\mathrm{e}}$ pylône et sur les fondations occidentales d'obélisques situées

\footnotetext{
* Chargé de recherche au CNRS, UMR 5140 « Archéologie des sociétés méditerranéennes », équipe « Égypte nilotique et méditerranéenne », université Paul Valéry (Montpellier III)

${ }^{1} \mathrm{~J}$. YOYOTTE, « Un porche doré : La porte du IV pylône au grand temple de Karnak », CdE 28/5556 (1953), p. 28-38.

2 J. LECLANT, « Les inscriptions "éthiopiennes" sur la porte du IV ${ }^{e}$ pylône du grand temple d'Amon à Karnak », RdE 8 (1951), p. 101-120.

${ }^{3}$ P. BARGUET, Le temple d'Amon-Rê à Karnak. Essai d'exégèse, RAPH 21, Le Caire, 1962, p. 8796.
} 
dans les fondations des môles ${ }^{4}$. Les travaux de B. Letellier permirent ensuite de se faire une idée précise des parois et des portiques à piliers dont Thoutmosis IV avait paré le parvis du temple ${ }^{5}$. Les travaux du CFEETK dans ce secteur apportèrent leur lot de découvertes, dont les restes d'un colosse osiriaque de Sésostris III ${ }^{6}$. Je proposais moi-même une reconstitution de la cour de fêtes de Thoutmosis II et une hypothèse sur les obélisques prévus en son centre par Thoutmosis II, dressés par Hatchepsout et abattus par Amenhotep III ${ }^{7}$. A. Masson et M. Millet, enfin, effectuèrent en 2000-2001 des sondages devant le môle nord du IVe pylône, à la recherche, notamment, d'éventuelles traces des fondations des péristyles de Thoutmosis $\mathrm{IV}^{8}$.

1.2. L'histoire de l'évolution de ce secteur a fait l'objet d'un relatif consensus avec une reconstitution historique «standard » à laquelle j'ai adhéré et que j'ai, d'ailleurs, en partie alimentée. On peut la résumer comme suit : Thoutmosis Ier élève les Ve et IVe pylônes et les deux obélisques orientaux en façade de ce dernier, à la « Double Porte» du temple. Thoutmosis II fait construire la « cour de fêtes » pour circonscrire le parvis et projette de dresser en son centre les deux obélisques occidentaux. Ils seront extraits des carrières après son décès par Senenmout et dressés une fois Hatchepsout devenue roi et portent des mentions de Thoutmosis II et de la reine pharaon. Thoutmosis III dresse enfin dans la cour les deux obélisques médians, ainsi qu'un reposoir de barque en calcite. Thoutmosis IV orne la «cour de fêtes » de parois historiées, de péristyles, dispose un «porche » doré en façade du IVe pylône et aboute une chapelle de calcite à celle de son grand-père. Amenhotep III, après avoir dans un premier temps entrepris d'effectuer des aménagements et modifications de la « cour de fêtes », choisit la solution radicale d'implanter son IIIe pylône à l'emplacement des $2 / 3$ occidentaux du parvis. La paire occidentale d'obélisques de Thoutmosis II-Hatchepsout est sacrifiée ; les fragments entreposés ça et là, seront remployés plus tard et quelques uns sont peut-être inclus dans la maçonnerie du

${ }^{4}$ S. SAUNERON - J. VERITE, « Fouilles dans la zone axiale du III ${ }^{\mathrm{e}}$ pylône à Karnak », Kêmi 19 (= Les cahiers de Karnak II), 1969, p. 262-267 ; H. CHEVRIER, « Rapport sur les travaux de Karnak (1933-1934)», ASAE 34 (1934), p. 163.

${ }^{5}$ B. LETELLIER, « La cour à péristyle de Thoutmosis IV à Karnak », BSFE 84 (mars 1979), p. 33 49 ; « La cour à péristyle de Thoutmosis IV à Karnak (et la “cour de fêtes ” de Thoutmosis Il) » dans Hommages Sauneron I, BdE 81, Le Caire 1979, p. 51 à 71 ; ead. "Thoutmosis IV à Karnak : hommage tardif rendu à un bâtisseur malchanceux », BSFE 122 (1991), p. 36-52.

${ }^{6}$ J. LAUFFRAY, « Rapport sur les travaux de Karnak », Les cahiers de Karnak IV (= Kêmi 21, 1971), p. 62 ; B. LETELLIER, «Découverte d'une tête colossale de Sésostris III à Karnak », Les cahiers de Karnak IV (= Kêmi 21, 1971), p. 165-176 (tête) ; Le buste a été découvert ultérieurement par M. AZIM, voir A.H. MAAROUF - Th. ZIMMER, «Le Moyen-Empire à Karnak : varia 2 », Les cahiers de Karnak IX (1993), p. 232, § 6.

${ }^{7}$ L. GABOLDE, « À propos de deux obélisques de Thoutmosis II dédiés à son père Thoutmosis I et érigés sous le règne d'Hatchepsout-pharaon à l'ouest du IV pylône », Les cahiers de Karnak VIII (1988), p. 143-158 : id. "La "cour de fêtes" de Thoutmosis II à Karnak », Les cahiers de Karnak IX, 1993, p.1-99; id. "Compléments sur les obélisques et la "cour de fêtes" de Thoutmosis II à Karnak », Les cahiers de Karnak XI (2003), p. 417-468.

${ }^{8}$ A. Masson, M. Millet, «Sondage sur le parvis nord du IV e pylône », Les cahiers de Karnak XII/2 (2007), p. 659-680. 
nouveau pylône. Les dés de base sont transportés à Karnak-nord. La chapelle en calcite de Thoutmosis III et celle de Thoutmosis IV sont démontées et leurs fragments sont dispersés. Certains sont remployés dans les fondations du IIIe pylône avec les blocs de la chapelle de calcite d'Amenhotep Ier et ceux de la «chapelle blanche» de Sésostris Ier. Pendant l'hérésie amarnienne, les noms d'Amon sont martelés sur les obélisques de Thoutmosis Ier, sur ceux de Thoutmosis III (mais pas jusqu'en haut), sur les éléments divers des chapelles démantelées qui avaient été dispersés et non remployés. Les obélisques au nom de Thoutmosis II-Hatchepsout, abattus, échappent apparemment à la proscription d'Amon. À l'époque ramesside, des colonnes de texte sont ajoutées sur les obélisques de Thoutmosis Ier et ceux de Thoutmosis III.

1.3. Tout récemment Fr. Larché a proposé une vision différente de l'évolution architecturale de ce secteur, d'une part en remettant en cause la date de mise en place et l'attribution des obélisques du parvis du IVe pylône et, d'autre part, en proposant de replacer sur l'axe majeur du temple, entre les obélisques de Thoutmosis Ier, et le dos à l'entrée, la chapelle de calcite d'Amenhotep II $^{9}$.

Ces hypothèses novatrices et parfois surprenantes méritent qu'on les mette à l'épreuve des faits. Pour la clarté de la démonstration, on en donnera ici un résumé. Reprenant à son compte plusieurs éléments d'une hypothèse de Chr. Wallet Lebrun ${ }^{10}$, Fr. Larché fait les propositions suivantes :

1.3.1. Le IVe pylône aurait été édifié par Hatchepsout (et non Thoutmosis Ier comme on l'admet en général);

1.3.2. La «cour de fêtes» de Thoutmosis II serait posthume, œuvre encore d'Hatchepsout ;

1.3.3. Les obélisques orientaux du IVe pylône, extraits des carrières et décorés par Thoutmosis Ier, auraient été abandonnés gisants et dressés seulement par Hatchepsout sur les fondations orientales sises devant le IVe pylône.

1.3.4. Les fondations des obélisques de Thoutmosis Ier (paire orientale) et Thoutmosis III ${ }^{11}$ (paire médiane assignée par Fr. Larché à Thoutmosis II) auraient été réalisées conjointement en une seule étape de construction, par Hatchepsout.

1.3.5. Les obélisques au nom de Thoutmosis II (et, par endroit, d'Hatchepsout) auraient été dressés par Hatchepsout sur les fondations médianes dans la même phase de chantier (et non sur les bases occidentales comme on l'a proposé jusqu'ici).

1.3.6. Thoutmosis III aurait dressé une troisième paire d'obélisques sur les fondations occidentales indépendantes.

\footnotetext{
${ }^{9}$ Fr. LARCHE, « Nouvelles observations sur les monuments du Moyen et du Nouvel Empire dans la zone centrale du temple d'Amon », Les cahiers de Karnak XII/2 (2007), p. 407-592, sp. 469-480 et une version anglaise, très peu modifiée (avec la colaboration de Ch. VAN SICLEN) : id. "The Chapel of Amenhotep II Embedded between the Obelisks of Tuthmosis I », Les cahiers de Karnak XIII (2010), p. 297-326.

${ }_{10}$ Chr. Wallet-Lebrun, « Note sur le temple d'Amon-Rê à Karnak (I) », BIFAO 82 (1982), p. 355-362 ; ses thèses sont reprises dans Ead., Le grand livre de pierre, MAIBL 41, Paris, 2009, p. 6187.

${ }^{11}$ Selon la terminologie habituelle, à laquelle, on le verra, il faudra revenir.
} 
1.3.7. Amenhotep II aurait glissé sa chapelle de calcite entre les obélisques de Thoutmosis Ier, le dos à l'entrée ouest. La chapelle se serait donc ouverte à l'est. Elle aurait été abattue dès le règne de Thoutmosis IV.

1.3.8. Amenhotep III, à l'occasion de la construction du $\mathrm{III}^{\mathrm{e}}$ pylône, aurait abattu les obélisques occidentaux (supposés être ceux de Thoutmosis III) qui, entreposés ailleurs, auraient échappé aux martelages amarniens.

1.3.9. Amenhotep III aurait encore abattu les obélisques médians (supposés avoir été ceux aux noms de Thoutmosis II et Hatchepsout), lesquels se seraient brisés pendant la chute et dont certains tronçons auraient été inclus dans le remplissage du $\mathrm{III}^{\mathrm{e}}$ pylône.

1.3.10. À l'époque ramesside, à l'occasion d'un des jubilés de Ramsès II, on aurait replacé sur les bases médianes les obélisques que Thoutmosis III aurait, primitivement, dressés sur les deux bases situées le plus à l'ouest et qui avaient été incluses dans les fondations du $\mathrm{III}^{\mathrm{e}}$ pylône.

\section{Des biais dans les raisonnements}

2.1. Nous allons examiner les éléments de cette proposition, mais il est nécessaire au préalable de pointer deux biais récurrents dans les raisonnements qui nous sont proposés.

Le premier de ces biais consiste à considérer que nombre d'édifices, inscrits au nom d'un souverain précis, ont été en réalité bâtis par un autre. Il nous est suggéré, en la circonstance, qu'ils sont posthumes et dédiés par un pharaon à un de ses prédécesseurs: on nous propose ainsi que le $\mathrm{IV}^{\mathrm{e}}$ pylône soit l'œuvre d'Hatchepsout, dédié à titre posthume à Thoutmosis I ${ }^{\text {er }}$ :

« Hatchepsout fit construire au nom de Thoutmosis Ir le IV pylône avec sa clôture et ses pièces latérales $[\ldots]$. Ces monuments sont tous au nom de son père défunt comme le montrent la décoration du pylône et de ses niches, aussi bien que les inscriptions sur les colosses osiriaques qui y sont adossés ${ }^{12}$.

Il est encore suggéré, nous l'avons vu, que la cour de fête de Thoutmosis II soit aussi une œuvre posthume, réalisée par Hatchepsout.

Si l'existence de réalisations posthumes est avérée, il s'agit de cas rares dans des conjonctures très particulières. On sait par exemple que le Netery-menou au nom de Thoutmosis II comportait des représentations posthumes du roi, mais elles sont accompagnées dans le même édifice de nombreuses scènes avec le nom de Thoutmosis III, de palimpsestes très repérables, de titres comme celui de «sœur de roi » attribué à Néferourê, de l'omniprésence de la régente Hatchepsout, qui sont autant d'indices permettant de déceler la nature posthume de certains reliefs. Celle-ci s'expliquait, en outre, par les circonstances particulières entourant le décès inopiné de Thoutmosis II, la jeunesse de Thoutmosis III à son avènement et l'instauration nécessaire d'une régence ${ }^{13}$.

En dehors des exceptions de ce type, rares, justifiées et aisément décelables, les pharaons construisent toujours en leur nom et non en celui de leurs prédécesseurs. C'est même leur devoir et leur privilège. On se méprendrait gravement sur la mentalité des Anciens en croyant que des rois peuvent

${ }^{12}$ Fr. LARCHE, « Nouvelles observations ... », p. 492.

${ }^{13} \mathrm{~L}$. GABOLDE, Monuments décorés en bas-reliefs, aux noms de Thoutmosis II et Hatchepsout à Karnak, MIFAO 123, Le Caire, 2005, p. 13-17. 
naturellement réaliser un édifice intégralement au nom d'un ancêtre et s'effacer au point de ne pas s'y faire mentionner. En l'absence d'indices probants il faut donc se dispenser de recourir à ce genre d'hypothèse.

2.2. Le second biais est une sorte de commodité dont il est fait usage à l'excès consistant à considérer que la réalisation d'un monument s'est étirée d'un règne à l'autre (voire sur plusieurs règnes) ce qui permet, selon les besoins, de rattacher l'édifice tantôt à l'un ou tantôt à l'autre de ces règnes. Si le cas peut se présenter occasionnellement (obélisque du Latran par exemple), ce n'est certainement pas la règle et ce genre de pratique doit être considéré comme une exception rare. Thoutmosis IV, du reste, signe dûment l'achèvement de l'obélisque du Latran par ses propres dédicaces. On nous propose ainsi que les obélisques de Thoutmosis Ier aient été en projet sous le règne de ce pharaon, soit devant le $\mathrm{V}^{\mathrm{e}}$ pylône, soit devant un $\mathrm{IV}^{\mathrm{e}}$ pylône encore lui-même en projet et que leur mise en place aurait attendu l'avènement d'Hatchepsout-pharaon : aucun indice ne vient appuyer cette supposition et Hatchepsout n'ajoute aucune dédicace alors que la place ne manquait pas sur les obélisques comme l'ont prouvé les Ramsès qui ont par la suite ajouté leurs propres colonnes de texte.

\section{La date du IV pylône.}

3.1. Les encadrements des niches de la face orientale du IV IV $^{\mathrm{e}}$ pyône portent clairement le nom de Thoutmosis $\mathrm{I}^{\mathrm{er}}-h^{\mathrm{c}}-n f r w^{14}$ (fig. 1 et pl. I.a).

3.2. Les colosses osiriaques qui sont répartis sur le pourtour de la salle, ajoutés vraisemblablement dans une seconde phase de travaux, notamment contre le IV $^{\mathrm{e}}$ pylône, sont tous au nom de Thoutmosis $\mathrm{I}^{\mathrm{er}}$ (vivant) ${ }^{15}$.

3.3. De même, les piles qui sont aux deux extrémités nord et sud de la salle, appuyées contre les murs qui font corps avec le IV pylône, sont-elles, elles aussi, au nom original de Thoutmosis $\mathrm{I}^{\mathrm{er}}$ (vivant) ${ }^{16}$ (pl. II, a-b).

3.4. Le linteau de la porte menant à 1 'escalier du $\mathrm{IV}^{\mathrm{e}}$ pylône est lui aussi au nom de Thoutmosis I $^{\text {er }}$ (vivant) ${ }^{17}$ (pl. II, c) :

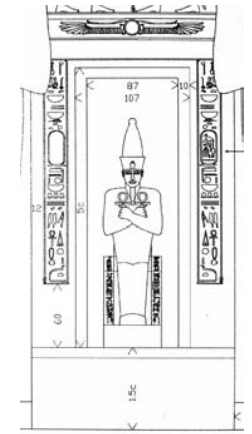

Fig. 1. Niche au nom de Thoutmosis I ${ }^{\text {er }}$ face est du IVe pylône (d'après Fr. Larché, Karnak XII pl. XLVIII)

3.5. Le $I^{\mathrm{e}}$ pylône est donc, dans ses œuvres, entièrement au nom de Thoutmosis I $^{\text {er }}$ (vivant). En conséquence de quoi, il n'y a aucune raison de supposer qu'il a été bâti par un autre souverain. À l'inverse, lorsque Hatchepsout dédie à son père des statues (éléments mobiliers) et les place dans les niches orientales de la partie nord de la Ouadjyt, elle les pourvoit d'une dédicace où son

\footnotetext{
${ }^{14}$ Fr. LARCHE, « Nouvelles observations ... », pl. XLVIII.

15 J.-Fr. CARLOTTI - L. GABOLDE, « Nouvelles données sur la Ouadjyt », Les cahiers de Karnak XI (2003), p. 268-269, n 4.

16 J.-Fr. CARLOTTI - L. GABOLDE, « Nouvelles données sur la Ouadjyt », p. 269, n 5.

17 J.-Fr. CARlotti - L. Gabolde, « Nouvelles données sur la Ouadjyt », n 6 et P. Barguet. Le temple d'Amon-Rê, p. 97.
} 
nom est, cette fois-ci, bien présent, à côté de celui de son père (mentionné en dédicataire) ${ }^{18}$.

3.6. Le $\mathrm{IV}^{\mathrm{e}}$ pylône a été construit en même temps que le $\mathrm{V}^{\mathrm{e}}$ pylône sur un radier continu et établi à une même hauteur ${ }^{19}$. Les murs qui viennent s'abouter à la Ouadjyt aux angles nord-est et sud-est sont manifestement plus tardifs : d'une part une différence nette d'assisage et un coup de sabre ont été repérés en fondation et, d'autre part, ils reposent sur des fondations dont l'assise supérieure a un lit d'attente notoirement plus élevé (au nord, il est au moins $11 \mathrm{~cm}$ plus haut ${ }^{20}$, et au sud, de 12 à $14 \mathrm{~cm}$ plus haut ${ }^{21}$ ); le mur de péribole partant vers l'est des angles nord-est et sud-est de la Ouadjyt est donc nécessairement postérieur à la Ouadjyt elle-même (fig. 2). À l'inverse du côté ouest, les assises de fondations se succèdent à une même hauteur au niveau du lit d'attente, pour le $\mathrm{V}^{\mathrm{e}}$ pylône, les murs de la Ouadjyt et le $\mathrm{IV}^{\mathrm{e}}$ pylône (avec toutefois un très léger pendage général du nord vers le sud, semble-t-il).

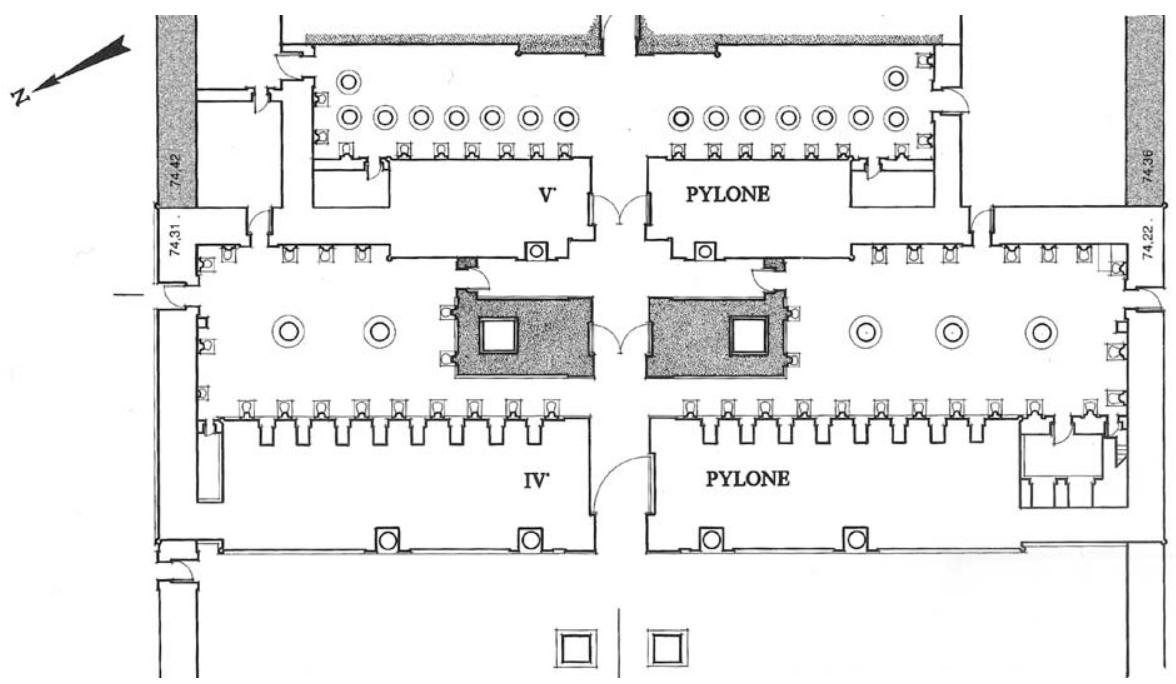

Fig. 2. Chronologie des murs aboutés à l'est de la Ouadjyt, confirmée par la différence de niveau des assises de fondation (dessins J.-Fr. Carlotti).

3.7. Ces deux pylônes (les $\mathrm{IV}^{\mathrm{e}}$ et $\mathrm{V}^{\mathrm{e}}$ ) sont, par ailleurs, mentionnés par Inéni dans sa biographie :

\footnotetext{
${ }^{18}$ Fr. LARCHE, « Nouvelles observations ... », pl. LII-LIV.

19 J.-Fr. CARLOTTI - L. GABOLDE, « Nouvelles données sur la Ouadjyt », p. 255-258.

$2074,42 \mathrm{~m}-74,31 \mathrm{~m}=11 \mathrm{~cm}$ : J.-Fr. CARLOTTI - L. GABOLDE, « Nouvelles données sur la Ouadjyt», p. 256. Curieusement Fr. Larché ne tient aucun compte, dans ses reconstitutions chronologiques, de cette importante différence de niveau des lits d'attente des assises supérieures des fondations.

21 74,36 m -74,22 m = $14 \mathrm{~cm}:$ M. AZIM et alii, Karnak et sa topographie I, Paris, 1998, pl. 3.
} 


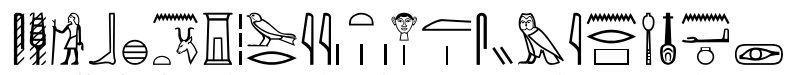

«j'[ai érigé] de grands pylônes sur ses deux côtés (= ceux de la iounyt) en belle pierre blanche de calcaire ${ }^{22}$.

Ils sont ensuite cités dans les textes d'Hatchepsout qui rapportent qu'elle fait dresser ses obélisques (prévus en électrum et réalisés en granit) «dans l'intervalle des deux grands pylônes du roi taureau victorieux, roi de Haute et Basse-Égypte Âakheperkarê, juste de voix » ${ }^{23}$ et, surtout, dans le récit gravé sur les blocs nouvellement trouvés des annales de Thoutmosis III ${ }^{24}$. Or, à l'époque de Thoutmosis III, il ne peut en aucune manière s'agir d'autre chose que des IV et $\mathrm{V}^{\mathrm{e}}$ pylônes ${ }^{25}$.

3.8. Les murs de la «cour de fêtes » de Thoutmosis II (qui est l'œuvre de ce roi voir infra, § suivant) s'appuient, aussi bien en fondation qu'au sommet, sur les murs qui prolongent le $\mathrm{IV}^{\mathrm{e}}$ pylône et qui font corps avec lui : la «cour de fêtes » de Thoutmosis II est donc bien postérieure au IV pylône $^{26}$.

3.9. Dès lors l'attribution du $\mathrm{IV}^{\mathrm{e}}$ pylône à Thoutmosis $\mathrm{I}^{\mathrm{er}}$ demeure la seule solution qui permette de rendre compte de manière satisfaisante de tous les points exposés ci-dessus.

\section{La « cour de fêtes » de Thoutmosis II}

4.1. Les bandeaux de dédicace de la «cour de fêtes » de Thoutmosis II sont intégralement à son nom, qu'il s'agisse des inscriptions dédicatoires des murs, des môles du pylône ou de celles des portes $\mathrm{A} \mathrm{r}^{\circ}-\mathrm{v}^{\circ}$, ou des portes $\mathrm{C}$ et $\mathrm{D}^{27}$. Aucun autre souverain n'y est mentionné. Il n'y a donc aucune raison de postuler que la «cour de fêtes » aurait été dressée par Hatchepsout qui signe toujours dûment ses réalisations ${ }^{28}$ : elle a bien été construite par celui qui déclare qu'il l'a fait personnellement ériger sur l'inscription dédicatoire ${ }^{29}$.

${ }^{22}$ Urk. IV 56,1-2. Les pylônes sont constitués en réalité d'un noyau en grès enveloppé d'un épais revêtement de calcaire.

${ }_{23}$ Urk. IV, 365, 3-5.

${ }^{24}$ N. GRIMAL, dans Annuaire du Collège de France 2005-2006, 2006, p. 583-584, fragment VII M. Dans le commentaire p. 587, de la ligne $(x+6)$, il est suggéré que Thoutmosis III aurait fait des aménagements entre les môles du IV $\mathrm{V}^{\mathrm{e}}$ pylône : on n'a la trace d'aucune intervention de Thoutmosis III sur la porte, tandis que ses aménagements dans la Ouadjyt entre les deux pylônes est, elle, en revanche, avérée.

${ }^{25} \mathrm{Le} \mathrm{VI}^{\mathrm{e}}$ pylône date d'Hatchepsout-Thoutmosis III : R. MENSAN dans F. BURGOS - Fr. LARCHE, La chapelle rouge II, ERC, Paris, 2008, p. 134-135 et 260-277.

${ }^{26}$ L. GABOLDE - T. ZIMMER, «Sondage effectué à l'angle sud-est du parvis du IV IV $^{\mathrm{e}}$ pône », $L a$ cahiers de Karnak VIII (1987), p. 161 ; L. GABOLDE, « La "cour de fêtes" de Thoutmosis II ... », p. 29.

${ }^{27}$ id. ibid., p. 30-44.

${ }^{28} \mathrm{~J}$ 'avais très hypothétiquement rattaché à la « cour de fêtes » des éléments d'une porte en calcaire aux noms de Thoutomsis III et d'Hatchepsout, qui n'avaient d'ailleurs pas (et n'ont toujours pas) de raccord direct avec le reste l'édifice (id. ibid., p. 52-53). Quand bien même il s'agirait d'un élément de décor de cet édifice, il serait plus logique d'y voir un achèvement très modeste et ponctuel du bâtiment par les successeurs de Thoutmosis II.

${ }^{29}$ L. GABOLDE, «La "cour de fêtes” de Thoutmosis II à Karnak », p. 34 et pl. 9. Je ne connais du reste pas d'inscription dédicatoire posthume, sauf quand il s'agit d'une restauration du texte, sur le monument même construit par le souverain (comme c'est le cas ici ou par exemple, avec la dédicace 


\section{Les obélisques de Thoutmosis $\mathrm{I}^{\mathrm{er}}$}

5.1. Les obélisques de Thoutmosis $\mathrm{I}^{\mathrm{er}}$ portent une dédicace de ce roi mentionnant l'emplacement où ils doivent être dressés, selon les termes même leur initiateur:

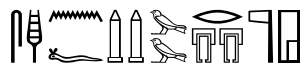

$s^{\prime} h^{\prime} n f \underline{t}$ thnwy wrwy r rwty hwt-nt $\underline{t}$,

«ériger pour lui (Amon) deux obélisques à la "Double-Porte" du temple » 30

Cet emplacement est bien connu : c'est le même que celui qui est désigné sur les représentations des obélisques de Thoutmosis III de la «cour de fêtes» (quelles que soient les bases sur lesquelles ils avaient été dressés). Il s'agit du parvis du temple, circonscrit par la «cour de fêtes» de Thoutmosis II et le IV ${ }^{\mathrm{e}}$ pylône. Ce secteur était situé à la croisée des deux axes, nord-sud et est-ouest, et avait déjà été marqué à l'époque d'Amenhotep $\mathrm{I}^{\mathrm{er}}$ par la présence de deux portes monumentales ${ }^{31}$. On trouve la formule sur la représentation des obélisques de Thoutmosis III du mur des annales :

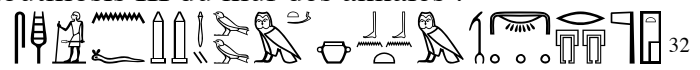

« ériger pour lui deux très grands obélisques en granit, les pyramidions étant en électrum, à la "Double-Porte" du temple »,

et sur les fragments des textes des annales nouvellement découverts et qui les mentionnent ${ }^{33}$. C'est donc bien sur le parvis du $\mathrm{IV}^{\mathrm{e}}$ pylône que Thoutmosis I ${ }^{\mathrm{er}}$ avait prévu de dresser ses obélisques.

5.2. La biographie d'Ineni nous enseigne d'ailleurs que Thoutmosis $I^{\mathrm{er}}$ a non seulement fait extraire les monolithes mais qu'il les a, effectivement, fait dresser sous la supervision de ce grand commis d'état :

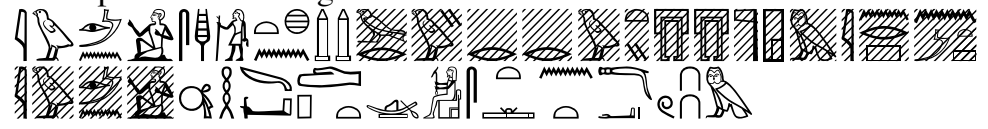

du sanctuaire central du temple d'Amenhotep III à Karnak-Nord, reconsacré à Montou à l'époque éthiopienne : L. GABOLDE - V. RONDOT, «Le temple de Montou n'était pas un temple à Montou », BSFE 136 (1996), p. 38, fig. 4.

${ }^{30}$ Urk. IV, 93,6.

31 Urk. IV , 43,4.

${ }^{32}$ Urk. IV, 642,12.

${ }^{33}$ N. GRIMAL, l.c. p. 592 et fragment VII N, col. $\mathrm{x}+6$. N. Grimal hésite curieusement entre les obélisques « en avant du temple » $\left(=\mathrm{du} \mathrm{IV}^{\mathrm{e}}\right.$ pylône $)$ et ceux de «l'axe sud » (= du VII $\mathrm{V}^{\mathrm{e}}$ pylône) alors que la mention de la « Double Porte » désigne nécessairement ceux du IV ${ }^{\mathrm{e}}$ pylône. 


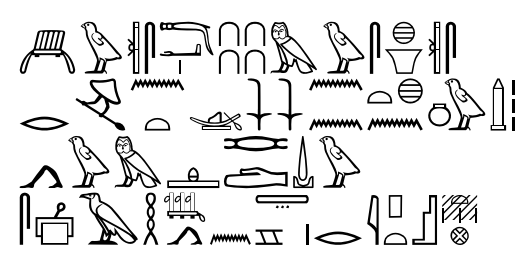

« J'ai supervisé l'érection de deux [grands] obélisques [...] [ayant ordonné] la taille d'une barque vénérable de cent-vingt coudées de longueur et de quarante coudées de largeur afin de faire naviguer ces deux obélisques, venus en paix (après avoir été) extraits (de la carrière) et ayant abordé à Karnak ${ }^{34}$.

Il n'y a évidemment aucune raison pour qu'Inéni se prévale dans sa biographie d'avoir lui-même supervisé l'érection des obélisques s'il ne l'a pas effectivement fait et si les monolithes sont restés couchés au sol ${ }^{35}$.

5.3. Dès lors, il n'y a pas d'autre solution que de considérer que les obélisques au nom de Thoutmosis Ier ont bien été dressés dès son règne et sur le

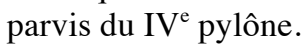

\section{La question des fondations des obélisques orientaux et médians et celle du supposé radier commun}

6.1. Les fondations des obélisques orientaux (Thoutmosis $\mathrm{I}^{\mathrm{er}}$ ) et celles des obélisques médians (Thoutmosis III) nous sont présentées comme constituant un radier unique construit d'un seul jet ${ }^{36}$. Elles n'ont pourtant aucune dalle particulièrement longitudinale - en commun : il n'y a aucun bloc qui aille d'une fondation à l'autre. Elles sont, de ce fait, parfaitement indépendantes. Il n'y a donc aucune raison de penser qu'elles constituent un seul radier. Les deux fondations ont été simplement juxtaposées (de manière assez lâche du reste) quand la seconde (la médiane) est venue s'abouter à la première (l'orientale). Deux pierres de calage situées entre les deux massifs à l'assise -2 ont été introduites là dans le processus chronologique naturel de mise en place des fondations médianes (pl. I,b) : après l'installation des dalles de l'assise -3, qui sont venues s'appuyer sur les fondations orientales, on a disposé des pierres de calage, l'une d'elles enfoncée de quelques cm sous le débord de l'assise -2, afin de renforcer la cohésion et la solidité de l'ensemble des fondations, et d'éviter de fragiliser l'assise des obélisques orientaux qu'on avait dû mettre à nu du côté ouest. Ensuite, on a mis en place les longues dalles longitudinales de l'assise -2.

\footnotetext{
${ }^{34}$ Urk. IV, 56, 10-16.

${ }^{35}$ Une biographie n'est pas une dédicace et on ne peut invoquer ici la dédicace de Thoutmosis III sur l'obélisque du Latran qu'il fallait bien graver au sol avant l'érection du monolithe, puisque l'obélisque est dressé avec ses quatre faces gravées. La dédicace est donc conforme au projet initial du roi, même s'il n'a pu le mener à son terme.

${ }^{36}$ Le nombre réel d'assises de fondation est inconnu et probablement plus important, les sondages n'ayant pu descendre sous la $3^{\mathrm{e}}$ assise en raison de la nappe phréatique (Fr. LARCHE, « Nouvelles observations ... », p. 474, n. 315).
} 


\section{Le processus se poursuivant ainsi jusqu'aux dalles de surface ${ }^{37}$ (voir les fig. 3 à}

$8)$.

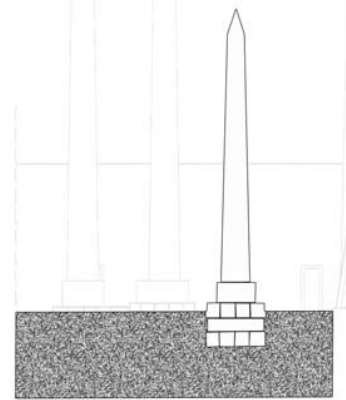

Fig. 3. Mise en place des obélisques devant le IV $^{\mathrm{e}}$ pylône : Phase I, Thoutmosis $\mathrm{I}^{\text {er }}$

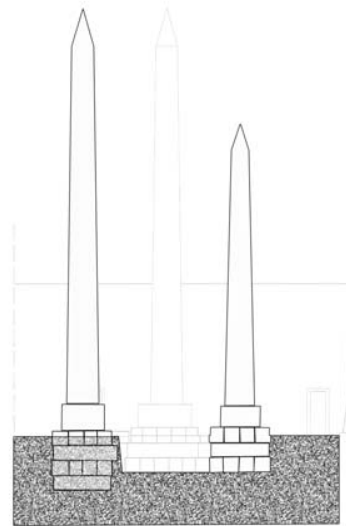

Fig. 6. Mise en place des obélisques devant le IV $^{\mathrm{e}}$ pylône : Phase III,1, Thoutmosis III : creusement de la fosse de fondation.

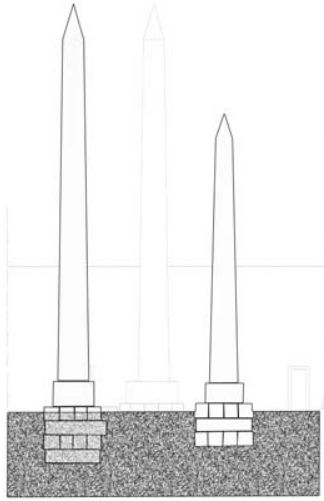

Fig. 4. Mise en place des obélisques devant le IV $^{\mathrm{e}}$ pylône : Phase II,1, Thoutmosis II-Hatchepsout fondations des obélisques occidentaux

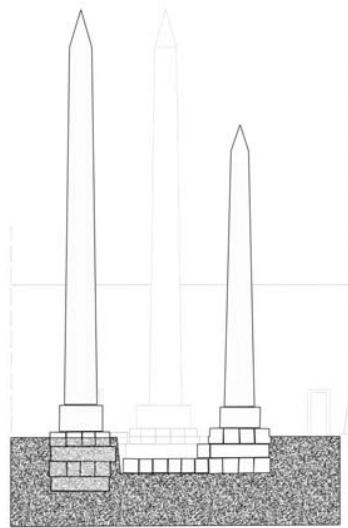

Fig. 7. Mise en place des obélisques devant le IV ${ }^{\mathrm{e}}$ pylône: Phase III,2, Thoutmosis III : mise en place des premières assises de fondation et des pierres de calage contre la fondation de Thoutmosis I ${ }^{\text {er }}$
Fig. 5. Mise en place des obélisques devant le $\mathrm{IV}^{\mathrm{e}}$ pylône : Phase II,2, Thoutmosis IIHatchepsout, remblaiement et mise en place du dé et des fûts

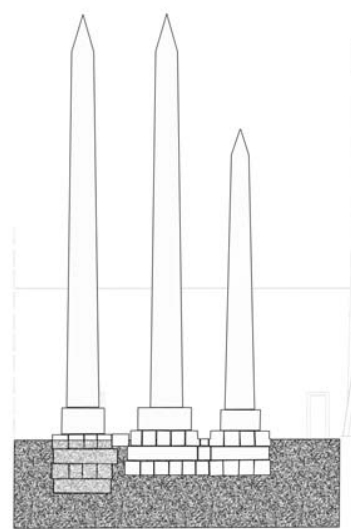

Fig. 8. Mise en place des obélisques devant le $\mathrm{IV}^{\mathrm{e}}$ pylône : Phase III,3, Thoutmosis III : achèvement des fondations, remblaiement et mise en place du dé de base et des monolithes.

${ }^{37}$ Je ne m'étends pas ici sur la disposition particulière des encoches ayant servi à accueillir les leviers en bois destinés à ajuster les dalles de l'assise supérieure de fondation, qui ne peut pas prouver grand chose. Ce que l'on peut en dire est qu'elles montrent, $1^{\circ}$ ) qu'il n'y avait rien à l'ouest (et au nord) des fondations de Thoutmosis $\mathrm{I}^{\text {er }}$ quand elles furent utilisées, $2^{\circ}$ ) qu'il n'y avait rien au nord et à l'ouest de l'obélisque médian de Thoutmosis III, ce qui est pleinement compatible avec la présence antérieure des obélisques occidentaux de Thoutmosis II puisque ceux-ci sont décalés vers le nord et vers l'ouest. 
6.2. Un des points curieux de l'hypothèse qui nous est proposée est que les obélisques médians (attribués par Fr. Larché à Thoutmosis II-Hatchepsout) auraient été placés si près et si complètement alignés sur le même axe que celui des obélisques orientaux au nom de Thoutmosis $\mathrm{I}^{\mathrm{er}}$ qu'ils auraient entièrement masqué ces derniers : on se demande vraiment pourquoi on n'avait pas tenté de palier cet inconvénient en décalant les obélisques médians pour que tous les monolithes soient visibles.

\section{Les obélisques médians (de Thoutmosis III)}

\section{et les obélisques occidentaux (de Thoutmosis II)}

7.1. Fr. Larché propose, nous l'avons vu, que les obélisques médians aient été ceux de Thoutmosis II-Hatchepsout et que les obélisques des fondations occidentales aient été ceux de Thoutmosis III.

Les obélisques au nom de Thoutmosis II et Hatchepsout ont été mis en chantier (ou peut-être simplement projetés) pendant les derniers mois du règne de ce souverain. Senenmout organisa semble-t-il leur extraction, pendant la régence d'Hatchepsout qui, devenue pharaon, y apposa son nom et les fit dresser ${ }^{38}$. Que les obélisques occidentaux aient été ceux de Thoutmosis II (et non ceux de Thoutmosis III) correspond à un raisonnement dont la logique avait été exposée dans les Cahiers de Karnak VIII ${ }^{39}$ et qui est la suivante: Thoutmosis II avait écarté ses obélisques (occidentaux) pour que ceux (orientaux) de son père Thoutmosis Ier demeurent visibles à l'arrière. Pour que les obélisques (médians) de Thoutmosis III demeurent visibles derrière ceux (occidentaux) de Thoutmosis II, il avait fallu les rapprocher de l'axe et consentir ainsi à masquer ceux (orientaux) de Thoutmosis Ier. De surcroît les obélisques de Thoutmosis II, abattus et sans doute en partie débités par Amenhotep III, avaient, sur l'un de leurs fûts au moins, un nom d'Amon qui avait, en raison même de cet abattage, échappé aux martelages amarniens ${ }^{40}$.

7.2. Les obélisques de Thoutmosis III montrent, en revanche, des traces manifestes de martelages du nom d'Amon, bien que ces martelages soient plus limités que de coutume et peu systématiques. Il est ainsi exact que les noms et figures d'Amon des tableaux de sommet de fût et les représentations du dieu sur les pyramidions des deux obélisques de Thoutmosis III semblent avoir été épargnés par les agents d'Akhénaton et c'est ce qui a induit Fr. Larché en erreur $^{41}$. Car les textes des fûts ne sont pas, eux, indemnes d'attaques atonistes et, malgré l'état très fragmentaire et dégradé des monolithes, quelques portions nous ont conservé des noms et épithètes d'Amon qui ont manifestement été martelés :

\footnotetext{
${ }^{38}$ L. GABOLDE, « À propos de deux obélisques de Thoutmosis II ... », p. 143-158.

${ }^{39}$ Id. ibid.

${ }^{40}$ Id, ibid., p. 147-148, fragment 13.

${ }^{41}$ Fr. LARCHE, « Nouvelles observations ... », p. 471 : «Les noms et figures d'Amon y sont intacts, preuve que les obélisques étaient invisibles sous Akhenaton alors que les autres obélisques de Thoutmosis III étaient visibles, ce qu'indiquent les martelages et les regravures sur l'obélisque unique (à Rome) et sur l'obélisque du VII pylône (à Istanbul)».
} 
- Obélisque sud, face nord, bloc $\mathrm{n}^{\circ} 232$. Ce bloc, qui fait partie d'un très long assemblage, montre un cartouche du nom de roi de Haute et Basse Égypte de Thoutmosis III, Mn-hpr-R` où celui-ci était pourvu de l'épithète tit Imn, « image d'Amon ». Le nom d'Amon a été clairement martelé (et regravé par la suite dans la dépression) (fig. 9)

- Obélisque sud, face ouest, bloc $\mathrm{n}^{\circ} 3$. Fragment de la dédicace : les signes $n s w t$ Ţ̌wy de l'épithète d'Amon ont, là encore, manifestement été martelés (et regravés par la suite dans la dépression) (fig. 10).

Fig. 9. Obélisque

de Thoutmosis III

du IV IV $^{\mathrm{e}}$ pyône,

monolithe sud, face nord, bloc $\mathrm{n}^{\circ} 232$.

En grisé :

martelage du nom d'Amon.

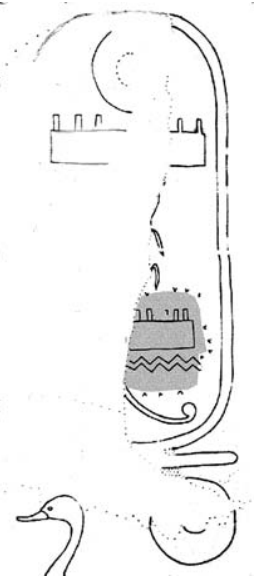

Fig. 10. Obélisque de Thoutmosis III du IVe pylône, monolithe sud, face ouest, bloc $n^{\circ} 3$. En grisé martelage de l'épithète d'Amon.

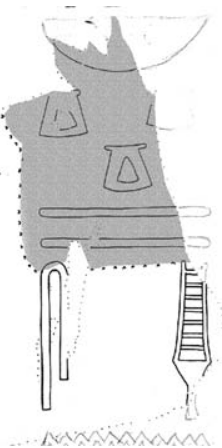

7.3. Dès lors, il faut comprendre que, pour une raison inconnue (état inachevé des figures ?), mais qui n'est certainement pas l'abattage des obélisques - lequel aurait, d'ailleurs, rendu certaines des images sommitales plus accessibles aux martelages - , les échafaudages d'Akhénaton ne s'élevèrent pas jusqu'au faîte des monolithes (comme s'ils n'avaient pas dépassé la hauteur des obélisques de Thoutmosis $I^{\mathrm{er}}$ ), ce qui épargna ainsi les figures hautes d'Amon ${ }^{42}$.

Il n'est donc plus nécessaire de supposer que les obélisques échappèrent à l'hérésie amarnienne parce qu'ils avaient été abattus : au contraire, ils étaient bel et bien accessibles et ont assurément subi des martelages, parce qu'ils étaient encore dressés pendant le règne d'Akhénaton.

7.4. Les éléments encore en place sur le dé de base nord des obélisques médians sont assurément des tronçons des obélisques de Thoutmosis III comme le montrent les assemblages préliminaires réalisés pour l'Atlas des obélisques de Karnak. Sur la partie basse des fûts se trouvent des scènes ajoutées par

42 Noter que si les représentations d'Amon sur les tableaux de sommet des fûts des obélisques d'Istanboul et du Latran ont bien été martelées par les agents d'Akhénaton, en revanche, les figures d'Amon sur toutes les faces des pyramidions de ces obélisques ont toutes été laissées intactes. De manière tout aussi étrange, les représentations d'Hatchepsout sont restées curieusement intouchées sur les parties hautes des obélisques de la Ouadjyt et sur leurs pyramidions (alors que les figures d'Amon furent, elles, attaquées jusqu'en haut, pyramidion compris). 
Ramsès II. Pour cette raison, Fr. Larché avait suggéré que ces obélisques - qu'il suppose avoir été dressés sur les fondations occidentales et abattus par Amenhotep III - auraient été redressés sur les fondations médianes (dont les obélisques, qui auraient été au nom de Thoutmosis II auraient été aussi abattus, au préalable, par le même Amenhotep III) à l'occasion d'un des jubilés de Ramsès II.

7.5. Il n'y avait, en fait, aucune nécessité pour Amenhotep III de procéder à l'abattage des obélisques médians. La mise à bas de tels monolithes est une opération difficile qui aurait été, d'ailleurs, très risquée, notamment dans la perspective d'une réutilisation ${ }^{43}$ et, finalement, totalement inutile puisque que les môles du III ${ }^{\mathrm{e}}$ pylône, englobant une partie des dés de base, venaient longer les obélisques à quelque distance des fûts. Les obélisques ne gênaient donc nullement la construction et il semble, au contraire, très clair qu'Amenhotep III avait fait en sorte de les épargner. La présence dès l'origine des obélisques de Thoutmosis III sur les bases médianes qu'ils n'auraient jamais quittées est donc la thèse qui rend le mieux et le plus simplement compte de la situation.

7.6. Le dessin de la tombe de Néferhotep est utilisé comme témoin pour estimer que la paire médiane d'obélisques aurait été abattue sous le règne d'Amenhotep III (fig. 11) :

La représentation de la tombe de Néferhotep ${ }^{44}$, comme la plupart des figurations antiques, n'est pas une reproduction fidèle de la réalité mais seulement une évocation de celle-ci. La présence d'un seul obélisque (valant pour une paire) là où deux (valant pour deux paires) auraient été requis ne doit pas surprendre: dans le cadre resserré de la figure, on a résumé l'essentiel en symbolisant avec un monolithe les quatre du secteur. D'ailleurs d'autres éléments que les obélisques, pourtant très volumineux ont été eux aussi « oubliés ». En effet, non seulement la maçonnerie de l'avant-porte en grès de Thoutmosis IV n'est pas figurée derrière le dais, mais surtout la colossale avant-porte en grès du III ${ }^{\mathrm{e}}$ pylône, décorée par

\footnotetext{
${ }^{43}$ Quelle que soit l'hypothèse envisagée pour leur emplacement primitif. C'est seulement avec la construction du temple d'Amon-Rê à Tanis que l'on a des indices de l'abattage d'obélisques (de $P i$ Ramsès ou d'ailleurs) et de leur ré-érection. À Karnak, le chemisage des obélisques de la Ouadjyt par Thoutmosis III, pour les dissimuler, semble montrer que les Égyptiens étaient réticents à mettre à bas de tels monolithes, voire incapables de le faire. Rappelons que lors de l'abattage de l'obélisque ouest du VII ${ }^{\mathrm{e}}$ pylône, a l'époque romaine, le monolithe se brisa au tiers de sa hauteur et seuls les $19 \mathrm{~m}$ supérieurs parvinrent à Istanboul (L. GABOLDE, «An Atlas of the Obelisks of Karnak », Egyptian Archaelogy 31, 2007, p. 33-35).

${ }^{44} \mathrm{PM} \mathrm{I}^{2}, 1$, p. 93, [15-16] ; N. de G. DAVIES, The Tomb of Nefer-hotep at Thebes I EEMM 9/1, New-York, 1933, p. 28-32 et pl. XLI. Sur cette scène, voir A. CABROL, Les voies processionnelles de Thèbes, p. 433-436. L'identification de la scène aux sanctuaires orientaux de Karnak, proposée il y a longtemps par Ch. DESROCHES-NOBLECOURT (« À propos de l'obélisque de Saint-Jean-de-Latran et d'un sanctuaire en vogue à Karnak à la fin de la XVIII ${ }^{\mathrm{e}}$ dynastie. Nouveaux exemples de scarabées commémoratifs de la XVIII ${ }^{\mathrm{e}}$ dynastie », ASAE 50 (1950), p. 257-267) n'a plus cours aujourd'hui : voir A. CABROL, «Remarques au sujet d'une scène de la tombe de Neferhotep (TT 49): Les fonctions de Neferhotep, la représentation des abords ouest de Karnak et son contexte », CRIPEL 15 (1993), p. 19-25. Conclusion similaire chez A. SCHLÜTER, Sakrale Architektur im Flachbild. Zum Realitätsbezug von Tempeldarstellungen, $\ddot{A} A T 78,2009$, p. 143-161.
} 
Amenhotep IV, n'a pas été représentée alors qu'il n'y a aucun doute sur son existence à l'époque de Néferhotep (règne d'Aÿ) ${ }^{45}$ et qu'elle présente une avancée de $10 \mathrm{~m}$ pour une hauteur de près de $18 \mathrm{~m}$; sans compter la maigreur proprement invraisemblable des pylônes. On note encore que les bases des obélisques médians, qui avaient été en partie englobées dans la maçonnerie du III ${ }^{\mathrm{e}}$ pylône (et donc n'ont jamais été enlevées), n'ont pas été figurées sur la fresque de la tombe de Néferhotep, pourtant supposée fidèle. À quoi auraient été utilisées ces bases devenues vides? À ce propos, on peut se demander pourquoi on ne les avait pas enlevées dans la foulée de l'abattage des obélisques ? Pourquoi les Ramsès auraient-ils redressés là les obélisques de Thoutmosis III, alors que l'espace était devenu exigu et qu'ils auraient été à peine visibles derrière le III $^{\mathrm{e}}$ pylône ? N'aurait-il pas été plus judicieux de les dresser en façade de leur réalisation majeure : le $\mathrm{II}^{\mathrm{e}}$ pylône précédant la salle hypostyle ?

On peut faire appel, pour illustrer la fidélité toute relative de la scène de la tombe de Néferhotep, à bien d'autres figurations architecturales tout autant imparfaites comme, par exemple, celle du VII ${ }^{\mathrm{e}}$ pylône dans les magasins nord de Thoutmosis III : là c'est le nombre de mâts qui est insuffisant puisqu'on n'en voit que deux là où il en avait quatre ${ }^{46}$. On peut encore comparer les représentations du pylône de Ramsès II orné de statues dans la cour du temple de Louxor aux

${ }^{45}$ Sur les étapes de construction de cette avant-porte et de la salle hypostyle, voir l'article à paraître de J.-Fr. CARLOTTI et Ph. MARTinEZ, «Un "château de millions d'années" d'époque ramesside : la grande salle hypostyle du temple d'Amon-Rê à Karnak», dans Chr. LEBLANC (éd.), Actes du colloques "les temples de million d'années et le pouvoir royal à Thèbes au Nouvel-Empire : science et nouvelles technologies appliquées à l'archéologie », Memnonia supplément $n^{\circ} 2,2011$, p. 119-146.

${ }^{46} \mathrm{PM} \mathrm{II}^{2}$, p. 104, (309) ; M. PILLET, « Deux représentations inédites de portes ornées de pylônes, à Karnak », BIFAO 38 (1939), p. 249, fig. 3. La présence des obélisques rend très problématique l'identification avec le petit pylône qui donne accès au reposoir de barque prônée par Pillet, car ce petit pylône est dépourvu d'obélisques . 
vestiges de statues retrouvés devant le monument: sur le bas-relief toutes les statues portent le pschent surmontant le némès tandis que certaines des statues debout représentent le roi coiffé de la seule couronne blanche ou du seul pschent $^{47}$

7.7. Pour conclure sur la série des trois paires d'obélisques du IV ${ }^{\mathrm{e}}$ pylône, il s'avère que la théorie alternative qui nous a été proposée présente tout un ensemble de défauts qui, accumulés lui enlèvent beaucoup de vraisemblance. Elle est ainsi fondée sur des postulats qui n'ont pas été vérifiés: les obélisques orientaux et médians ne reposent nullement sur un seul radier, mais sur deux fondations indépendantes juxtaposées. Elle est inutilement compliquée : il faudrait que des obélisques au nom d'un roi (Thoutmosis ${ }^{\mathrm{er}}$ ) aient été dressés par un autre pharaon (Hatchepsout); il faudrait que deux paires d'obélisques aient été abattus alors que l'abattage d'une seule paire était nécessaire ; il faudrait que des obélisques (ceux de Thoutmosis III) aient été abattus puis ré-érigés quelques mètres plus loin. Elle est contredite par les textes ou les faits: la biographie d'Inéni assure que les obélisques orientaux du $\mathrm{IV}^{\mathrm{e}}$ pylône ont bien été dressés sous Thoutmosis I ${ }^{\mathrm{er}}$; les martelages amarniens sont bien présents sur les obélisques de Thoutmosis III, et toutes les conséquences qui étaient tirées de leur absence supposée sont donc caduques. Enfin elle n'est pas nécessaire pour expliquer la succession des événements intervenus sur le parvis de $\mathrm{IV}^{\mathrm{e}}$ pylône, la théorie «standard » demeurant valide et notoirement plus simple ; elle est donc, à tous points de vue, préférable.

\section{L'emplacement de la chapelle en calcite d'Amenhotep II entre les obélisques.}

8.1. Fr. Larché, Fr. Burgos et Ch.C. van Siclen proposent que la chapelle en calcite au nom d'Amenhotep II ait été élevée sur l'axe majeur, entre les obélisques de Thoutmosis $\mathrm{I}^{\mathrm{er}}$. Cette suggestion soulève de nombreuses difficultés.

8.2. Observons pour commencer que, compte tenu de l'absence de la base de l'obélisque nord et de l'absence du monolithe lui-même, rien ne prouve que la chapelle aurait pu exactement tenir dans l'intervalle. Admettons le, néanmoins, à titre d'hypothèse.

8.3. Il n'y a aucun impératif liturgique à construire une chapelle à cet emplacement, lequel est, de surcroît, déroutant et sans parallèle ${ }^{48}$. Nous sommes, en effet, en présence d'une chapelle fermée au fond par une paroi et qui par conséquent n'aurait pas permis aux processions de la traverser: il aurait fallu abandonner la voie axiale pour la contourner, ce qui n'est certes pas impossible, mais inutilement compliqué.

\footnotetext{
$47 \mathrm{PM} \mathrm{II}^{2}$, p. 308, 30.

${ }^{48}$ On ne peut vraiment les comparer aux chapelles présentes sur les dromos, préfigurations de certains kiosques, comme on en trouve à Karnak-Nord : la chapelle d'Amenhotep III du dromos se trouve très loin du portail d'entrée. Voir A. CABROL, Les voies processionnelles de Thèbes, p. 9-13 et 499-504. Des traces d'une chapelle ont été repérées sur le dromos du temple de Thoutmosis III à Deir al-Bahari, mais il semble s'agir, d'après les restes de décor d'un reposoir de barque associé à une station d'accueil de statues royales (id., ibid., p. 552-53).
} 
8.4. Cette situation est d'autant plus surprenante que le souci constant et vérifié de tous les bâtisseurs du Nouvel-Empire a été d'élargir toujours plus les porches monumentaux en allant vers l'ouest en dégageant l'axe majeur et il y aurait un vrai paradoxe à venir embarrasser le passage avec un monument adventice.

8.5. Je ne m'étendrai pas sur la description et l'interprétation qui nous ont été proposées des divers décrochements des blocs de la chapelle de calcite ou retailles des soubassements des obélisques. Ils sont assez peu probants et, surtout, ne sont en aucune manière univoques : bien des découpes en gradins de l'assise supérieure des fondations s'expliqueraient, par exemple, bien mieux par des opérations successives de mises en place et de restaurations de dallages.

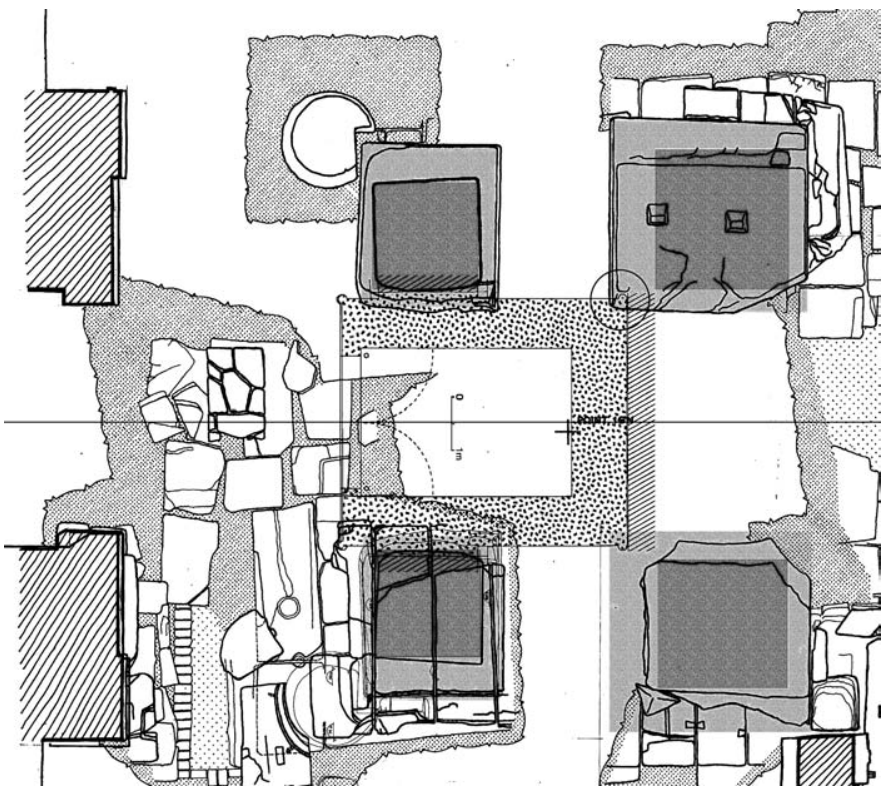

Fig. 12. Plan montrant l'hypothèse de la chapelle située entre les obélisques de Thoutmosis Ier :Le cercle souligne l'impossibilité de faire tenir la chapelle en raison de la présence de la base d'obélisque de Thoutmosis III (le Nord est en bas).

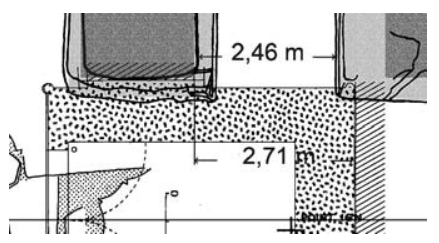

Fig. 13. Détail du plan montrant, mesures à l'appui, l'impossibilité de placer la chapelle de calcite entre les obélisques (le Nord est en bas).

8.6. La tentative de replacer en plan, sur le papier, la chapelle à l'emplacement proposé, en s'aidant de mesures de contrôle effectuées sur le terrain (fig. 12 et 13) montre clairement que la chapelle ne peut tenir là en raison de la présence de la base de l'obélisque sud de Thoutmosis III dont l'angle nord- 
est vient se superposer, sur une surface de $25 \mathrm{~cm}$ par $20 \mathrm{~cm}$, avec l'angle sudouest, bordé d'un tore, de la chapelle ${ }^{49}$.

8.7. Sous réserve de vérification plus approfondie, il semble bien, encore, que l'inclinaison en contrefruit des débords latéraux de la chapelle soit sensiblement plus forte que la pente réelle de l'obélisque de Thoutmosis Ier : si l'on ne voulait pas que la chapelle soit penchée, il aurait fallu combler un jour important à la partie basse du raccord.

8.8. La manœuvre de blocs aussi colossaux que ceux qu'utilise Amenhotep II, dans un espace aussi embarrassé par de multiples constructions antérieures (six obélisques et un pylône) aurait été extrêmement malaisée. Qu'on imagine la difficulté que pouvait représenter la mise en place des parois monolithes de la chapelle et, surtout, celle de sa dalle de couverture extrêmement pesante (86 tonnes selon Chevrier), ajustée de manière quasi jointive aux obélisques, ainsi qu'il nous est suggéré. Une erreur de poussée et la dalle était coincée ou les obélisques déstabilisés sur leurs assises. Le débord n'aurait d'ailleurs pas été une commodité en la circonstance mais, au contraire, un embarras. Il aurait été tellement plus simple et moins dangereux, en effet, d'avoir des parois planes sur les côtés de la chapelle et de combler l'espace entre celle-ci et les obélisques avec de discrets blocs rapportés.

Il est assez douteux que les Égyptiens anciens se soient ainsi compliqué la tâche sans véritable nécessité, alors que d'autres places étaient certainement disponibles pour installer la chapelle aux alentours .

8.9. Il est difficile enfin d'admettre qu'après que l'on ait réussi à ériger la chapelle, sans doute au prix de prouesses techniques extraordinaires, on ait résolu, quelques années seulement plus tard, sous le règne de Thoutmosis IV, de la démonter en affrontant, assurément, des difficultés et des risques de manœuvre équivalents, dans un espace toujours aussi contraint.

8.10. L'arrière de la chapelle est non décoré, sommairement épannelé et les tores sont rejetés sur les côtés. Cette disposition est en tous points identique à ce que l'on voit sur la face arrière de la chapelle adossée de Thoutmosis III à l'est ${ }^{50}$. Il est par ailleurs proprement impensable que la face du naos que l'on aurait découverte en pénétrant dans le temple, barrant l'axe majeur, ait été une grande paroi vide et à peine dégrossie .

8.11. Conscients de cette invraisemblance, Fr. Larché et Ch.C. van Siclen proposent de replacer sur cette paroi les éléments en granit rose d'Amenhotep II qui avait été découverts remployés dans ce secteur : un bloc célèbre représentant

\footnotetext{
${ }^{49}$ Les blocs de la partie basse de la chapelle de calcite semblent manquer, mais il n'y a aucune raison de penser que le tore ne descendait pas jusqu'au sol. On peut d'autant moins supposer une éventuelle retaille à la base de la chapelle que, du côté nord, un bloc d'angle, calé par une ligne de sol, descend assurément à 1,30 $\mathrm{m}$ du sol quand les socles font, eux, 1,67 $\mathrm{m}$ de haut. La base de granit sud de Thoutmosis III a été déplacée vers le sud puis remise en place par H. CHEVRIER lors de l'extraction du toit de la chapelle en calcite de Thoutmosis III ( « Rapport sur les travaux de Karnak (novembre 1926-mai 1927) », ASAE 27, 1927, p. 135-139 et fig. 1, p. 137). Il moins que probable que Chevrier ait pu se tromper lors de la remise en place.

${ }^{50} \mathrm{PM} \mathrm{II}^{2}$, p. 216, [18] ; P. BARGUET, Le temple d'Amon-Rê à Karnak, p. 220-221.
} 
Amenhotep II sur son char tirant des flèches sur une cible ${ }^{51}$ et deux blocs du musée du Caire, qui se raccordent, montrant Amenhotep II conduisant des prisonniers devant Amon ${ }^{52}$. La suggestion n'emporte pas la conviction. En effet, d'une part, on ne trouve aucune trace de mortaise au dos de la chapelle pour accrocher d'éventuelles dalles de décor rapportées et, d'autre part, les éléments décorés des musées du Caire et de Louxor, qui ne sont, de surcroît, pas centrés sur un axe, sont, eux, bien trop petits pour avoir pu occuper cet emplacement (fig. 14).

Fig. 14. Superposition à une même échelle des dalles en granit d'Amenhotep II et de la face arrière de la chapelle de calcite qui serait celle que l'on aurait découverte en entrant dans le temple.

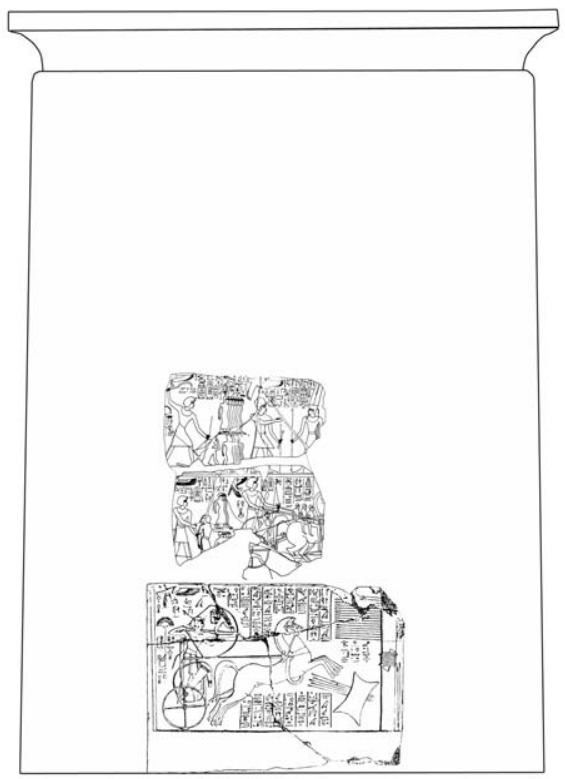

\section{Proposition alternative pour la chapelle d'Amenhotep II}

9.1. Que faire dès lors de la chapelle de calcite d'Amenhotep II si l'on récuse l'emplacement entre les obélisques de Thoutmosis Ier? Une proposition très attrayante a été faite par J. Fr. Carlotti dans les Hommages Goyon, en restituant la chapelle de calcite et celle en granit d'Amon de Pérounefer face à face dans la partie nord de la « cour de la cachette » $\left(\right.$ fig. 15) ${ }^{53}$. Fr. Larché et Ch.C. van Siclen

${ }^{51} \mathrm{PM} \mathrm{II}^{2}$, p. 79 ; B.v. BothMER, J.P. RoMANO, Catalogue du musée d'art égyptien ancien de Louxor, BdE 95, 1985, p. 35-36, n 88. Extrait du III ${ }^{\mathrm{e}}$ pylône.

$52 \mathrm{PM} \mathrm{II}^{2}$, p. 74 ; JE 36360 ; extraits des fondations de l'avant-porte du IV ${ }^{\mathrm{e}}$ pylône ; A.H. ZAYED, «Une représentation inédite des campagnes d'Amenhotep II », Melanges Gamal ed-Din Mokhtar I, $B d E$ 97/1, 1985, p. 5-17. Noter que l'échelle des scènes sur les fragments du Caire est sensiblement inférieure à celle du fragment de Louxor. Ils sont ici superposés à titre indicatif, en réalité le niveau restitué des sols s'oppose à un assemblage de ce genre.

53 J.-Fr. CARLOTTI, «Le "mur fantôme" de la "cour de la cachette" du temple d'Amon-Rê à Karnak », Hommages offerts au Pr. Jean-Claude Goyon pour son 70ème anniversaire, IFAO, BdE 143, 2008, p. 55-66. 
lui ont opposé quelques objections qui, en vérité, ne se sont révélées aucunement rédhibitoires :

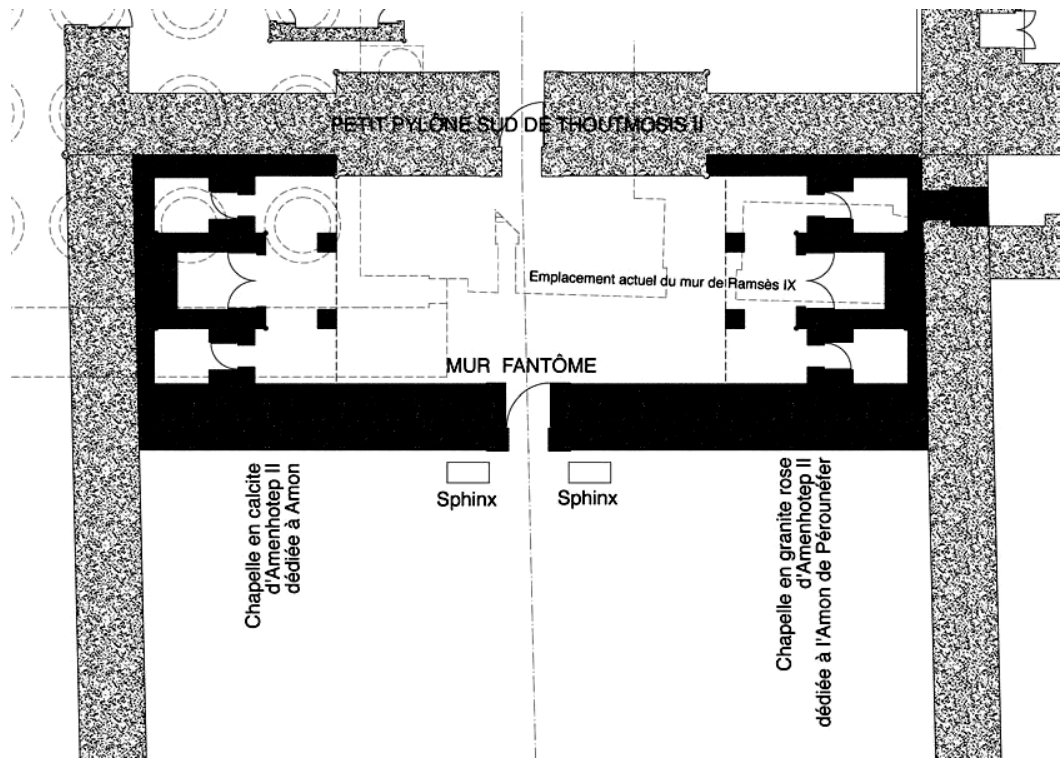

Fig. 15. Hypothèse de restitution de l'emplacement des chapelles de calcite et d'Amon de Pérounefer dans la « cour de la cachette (d'après. J. Fr. Carlotti, Hommages Goyon, p. 65, fig. 4)

9.2. La proportion donnée aux piliers est contestée ; pourtant, avec 6,00 m dans la restitution proposée par J.-Fr. Carlotti, ils ne sont pas particulièrement étirés lorsqu'on les compare à d'autres piliers d'Amenhotep II comme ceux du temple remonté entre les IXe et Xe pylônes qui atteignent, eux 5,20 m sous l'architrave. Mais, surtout, ces proportions, qui étaient purement indicatives, pourraient être parfaitement diminuées sans que cela change en rien la validité de l'hypothèse de restitution. Il en va de même pour la question discutée de la double couverture qui est très secondaire et sans impact sur la localisation de l'édifice.

9.3. Il est suggéré que des tambours de colonnes d'Amenhotep II mentionnant l'Amon de Pérounefer récemment identifiés remployés par Ramsès IV au temple de Khonsou soient des éléments des supports des architraves : c'est tout à fait possible, dans le cadre même de l'hypothèse de J.-Fr. Carlotti. Les colonnes polygonales auraient très bien pu être placées devant la chapelle en granit d'Amon de Pérounefer et des piliers carrés (ou d'autres colonnes polygonales) en face, devant la chapelle de calcite.

9.4. On a encore opposé à la restitution que la place disponible sur les architraves était insuffisante pour placer une dédicace symétrique autour d'un $f$ central. Avec un texte allant dans une seule direction, à savoir dans le sens de 
progression du roi (pratique très courante), on peut parfaitement développer la dédicace suivante sur les $10 \mathrm{~m}$ disponibles :

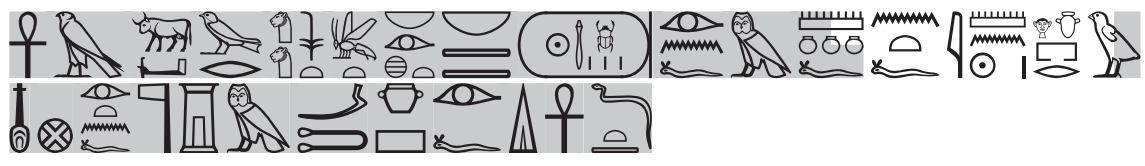

9.5. Fr. Larché propose qu'au lieu du «mur fantôme » il y ait eu une sorte de naos en bois au-dessus du criosphinx de la «cour de la cachette». Cette suggestion laisse perplexe car, malgré un examen très attentif d'une photo non surlignée, il ne m'a pas été possible de voir la trace potentielle du toit qu'il invoque à l'appui de sa suggestion, tandis que des murs à fruit pour former une sorte de naos en bois sont un peu surprenants (d'autant qu'ils sont d'épaisseur inégale). L'absence de trait de pose pour les parois intérieures du naos supposé est aussi anormale. En restituant un mur plein, cet obstacle disparaît.

9.6. On ne trouve, par ailleurs, aucune trace des trous de mortaises qui auraient été indispensables à la fixation du prétendu naos en bois sur le mur.

9.7. Une observation de logique peut encore guider le raisonnement: les scènes de Ramsès II s'arrêtent brusquement à gauche du tracé de pose et ce ne peut en aucune manière être en raison de la présence de la représentation de criosphinx puisque celle-ci est postérieure ${ }^{54}$ : la présence antérieure d'un mur fantôme à cet emplacement demeure bien la meilleure explication.

\section{Conclusion}

10.1. Les hypothèses alternatives - et souvent stimulantes - qui nous ont été proposées par Fr. Larché et Ch.C. van Siclen, peinent malgré tout à convaincre en raison de l'extrême complication qu'elles génèrent : elles nécessitent des monuments réalisés par d'autres que ceux qui les signent, des abattages d'obélisques (et leur ré-érection) superflus, des interprétations forcées de massifs de fondation. Elles supposent la construction d'une chapelle dans un site plutôt improbable et particulièrement peu approprié en raison du bâti existant. Elles ne sont nécessaires, ni sur le plan historique, ni sur le plan archéologique ni sur le plan liturgique et tout un éventail de solutions alternatives, plus simples, peuvent être proposées.

10.2. La théorie standard est ainsi moins compliquée et plus cohérente; elle demeure donc, à mes yeux, plus vraisemblable et donc préférable, toutes choses étant égales, en raison du très pertinent principe de parcimonie. Elle trouve un prolongement approprié dans la suggestion pleinement compatible de J.-Fr. Carlotti selon laquelle Amenhotep II aurait fait bâtir deux chapelles symétriques en calcite et en granit rose disposées face à face dans la partie nord de la « cour

\footnotetext{
${ }^{54}$ Merenptah à l'origine, martelé (par Amenmès) et apparemment non remplacé : voir P. BRAND, «Usurped Cartouches of Merenptah at Karnak and Luxor», in P. BRAND, L. COOPER (éd.), Causing his Name to live. Studies in Egyptian Epigraphy and History in Memory of William J. Murnane, Culture and History of the Ancient Near East 37, J. Brill, Leyde, 2009, p. 11 et fig. 10-11, p. 17-18.
} 
de la cachette ». La chapelle de calcite aurait disparu avec la construction du IIIe pylône tandis que celle d'Amon de Pérounefer et le mur «fantôme» de la cour de la cachette auraient été démontés ultérieurement et en plusieurs étapes. C'est aux savants de faire désormais leur choix entre les thèses en présence. 
PI. 1a. Niches au nom de Thoutmosis Ier face est du IVe pylône (d'après Fr. Larché, "Nouvelles observations ...», pl. XLVIII) ;
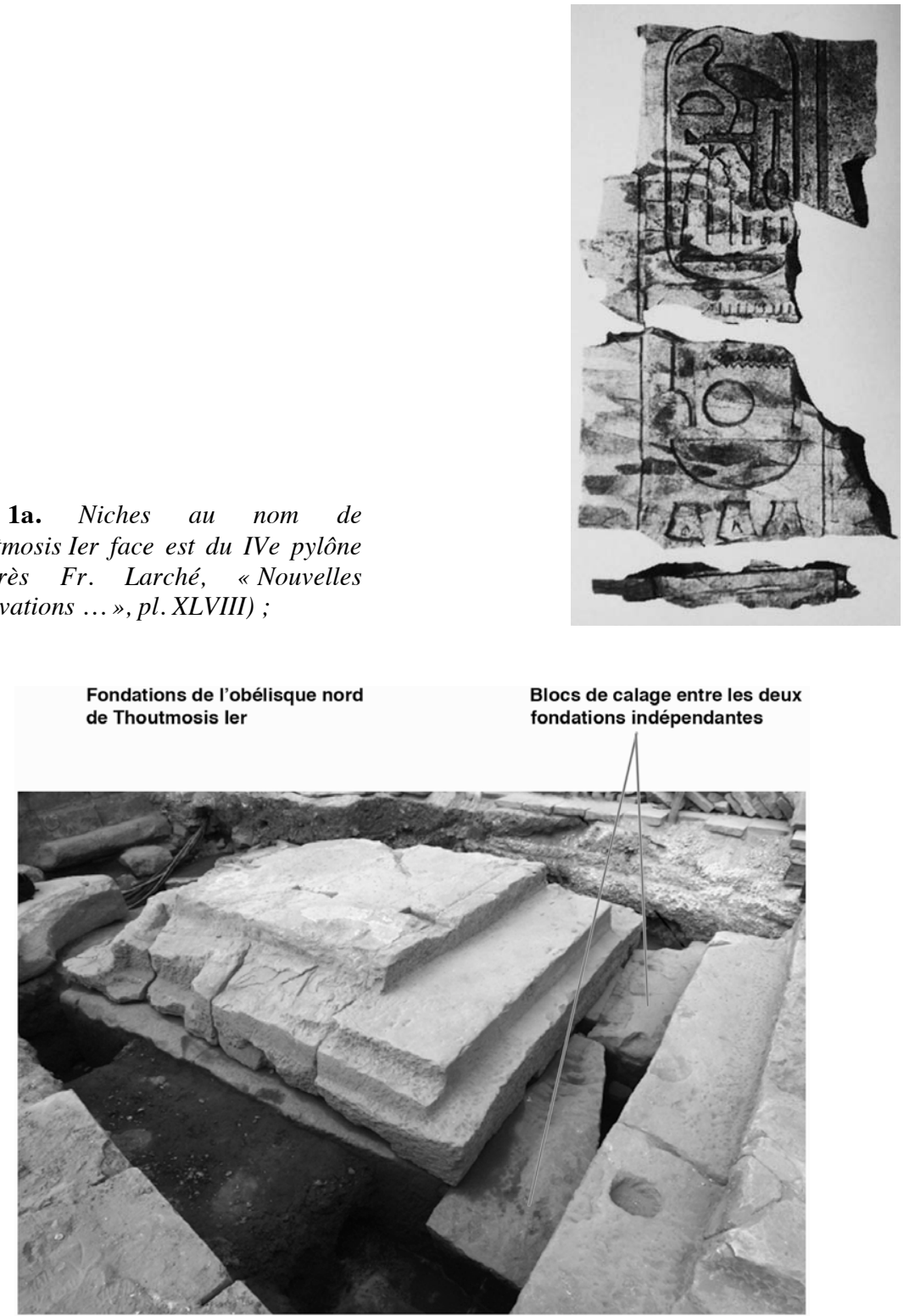

Fondations de l'obélisque nord de Thoutmosis III

PI. 1b. Les fondations nord, indépendantes, des obélisques orientaux (Thoutmosis Ier) et médians (Thoutmosis III) et les deux blocs de calages placés lors de l'installation de l'assise -2 (d'après Fr. Larché, Karnak XIII, fig. p. 316, - cliché A. Chéné. CFEETK) 


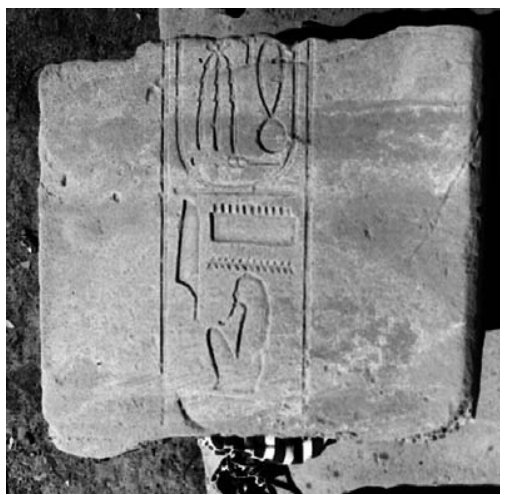

Pl. 2a. Piles de Thoutmosis $I^{e r}$ dans la Ouadjyt, phase II (photo J.-Fr. Carlotti - CFEETK)

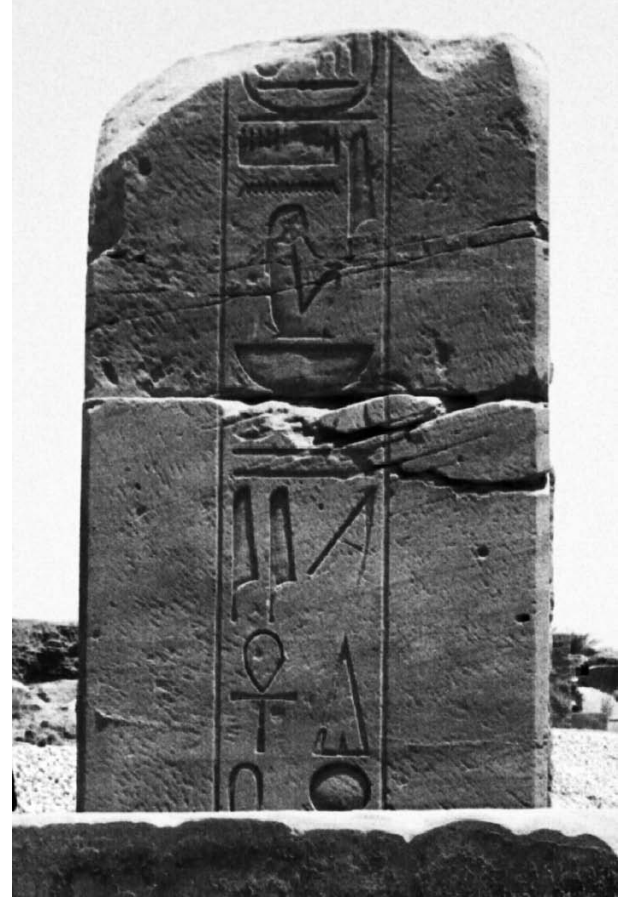

Pl. 2b. Piles de Thoutmosis $I^{\text {er }}$ dans la Ouadjyt, phase II (photo J.-Fr. Carlotti - CFEETK)

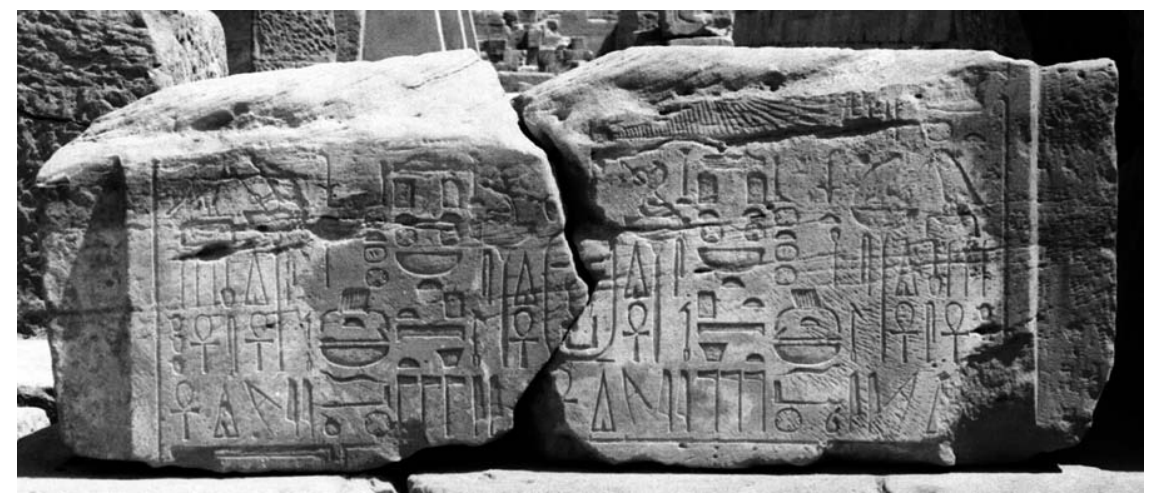

$2 c$

Pl. 2c Linteau de la porte de l'escalier d'accès aux môles du IVe pylône au nom de Thoutmosis I ${ }^{\text {er }}$ (photo J.-Fr. Carlotti - CFEETK). 